\title{
Design Circuit for the single-lens high-definition imaging system
}

\author{
Xiang Ren ${ }^{a}$,Yule Huang ${ }^{b}$, Chijun Li $^{c}$, Kai Yang ${ }^{d}$, Jiebin Zou ${ }^{e}$,Jiayong Kong ${ }^{\dagger}$, \\ Jing Cai*h
}

School of Physics and Optoelectronic Engineering, Foshan University, Foshan 528000, China;

a1595432290@qq.com, ${ }^{\text {b } 758407680 @ q q . c o m},{ }^{\mathrm{c}}$ 616121198@qq.com, ${ }^{\mathrm{d}} 810583915 @ q q c o m,{ }^{e} 272976$ 0005@qq.com,, $812295486 @ q q c o m$

*hCorresponding author: 476916176@qq.com

keyword: Chromatism ; High-definition imaging system ; Single lens ; Double light

Abstract: We proposed a method to resolve the problem of optical system defocus phenomenon by using the chromatic aberration of the single lens optical imaging system through reverse thinking. Furthermore, we designed and built the production which use SDRAM to collect data for CMOS image information literacy which was connected to the computer via USB transmission and the self-made circuit based on FPGA. Finally, some high-definition images can be obtained by using LabVIEW and MATLAB software programming to realize the data conversion, function calls, and image processing. The production is referred to the camera imaging system, specially, it can involves the problem which needs to solve the single lens of the depth of field and expand the depth of field in imaging system.

\section{Introduction}

Because of the need of using the shallow depth of field in macro photography, we cannot take photos for two or more objects clearly. Usually, in the design of photoelectric imaging system, because of the limitation of the optical elements in the system, the size of the depth of field also will be limited. Small depth of field in photoelectric imaging system cause we cannot take clear photos for two or more objects one-time.

There are many types of existing solution to the depth of field, can be roughly divided into two classes. The first is by increasing the depth of field to solve the problem of depth of field, such as spatial filter method, the optics apodization, wavefront coding and light sword optical element, etc. But the above methods all have in common is the technology is relatively complex and difficult to fit and part of the technology will increase accompanied by sacrifice or cost of image quality. The second category were taken according to the practical problems through many times focus or multiple sensor, along with depth of field synthesis technology (focus stacking) to solve the problem of depth of field. But as a result of operation will bring inconvenience to many focus or a lot and synthetic photos need to spend a lot of time.

To solve the single large depth of field lens imaging system from the focal problems in the process of filming, we can according to the actual situation, provides a simple and highly effective (and easy to learn) to solve in the design of photoelectric imaging system and macro photography in two or more subject to the depth of field problem. 


\section{Principle operation and experimental setup}

As is shown in Fig. 1, the design of the device is mainly built from a single lens color difference phenomenon existing in the imaging system, the method of using different wavelength light source illuminate the depth of field objects to chromatic aberration compensation system, from the focal problems, so that objects of different depth of field in like as clear as the same position. Device is divided into three parts: the light path, circuit part and software part.

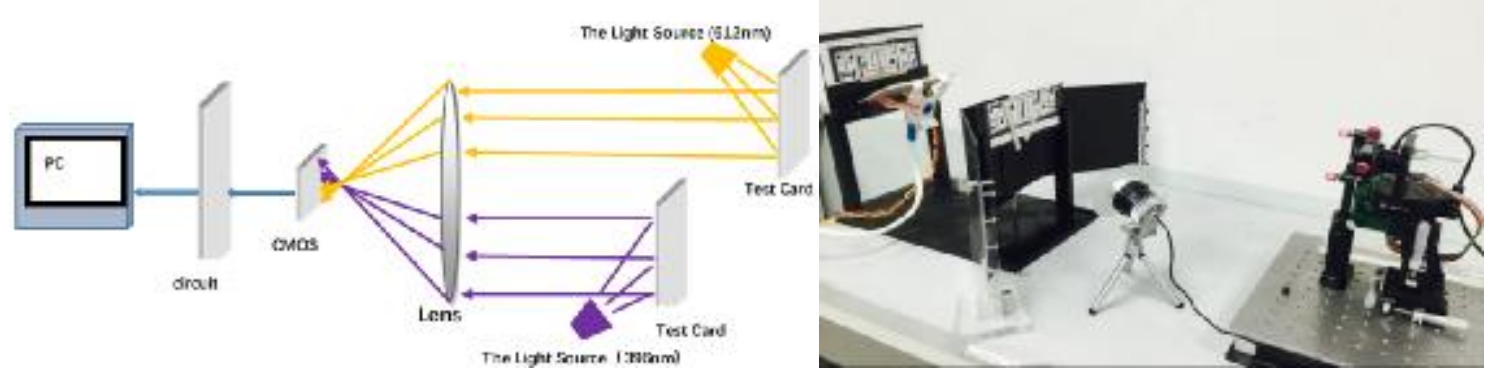

Fig. 1 overall plan diagram

Fig. 2 Real pictures

(1)Firstly, in aspect of light path. Light path portion of the building design is the use of single lens color difference in the formation of the principle of imaging system, through reverse thinking and use of different wavelengths of light source produce off color, in a certain depth range of several objects in the same position as party into clear like, while away from the focal problem, eliminates the position well color difference influence on imaging quality [1,2]. One way to determine the wavelength of light wave is to use the following formula

$$
\frac{1}{11}+\frac{1}{v}=\frac{1}{f}(1)
$$

( $\mathrm{f}$ is the focal length, $\mathrm{u}$ is the object distance, that is, subject to the lens, $v$ is image distance, the distance of the lens to the photographic device) respectively,[3] the focus of $500 \mathrm{~mm}$ test card surface is $37.6344086 \mathrm{~mm}, 800 \mathrm{~mm}$ in the focus plane of the test card is $36.60130719 \mathrm{~mm}$. Get the difference between the two test card like a distance of $1.03310141 \mathrm{~mm}$ (that is $v^{\prime}=1.03310141 \mathrm{~mm}$ )

Take $\lambda_{1}=656.3 \mathrm{~nm}$ wavelength in the index of refraction of $\mathrm{K} 9$ glass $\mathrm{n}_{1}=1.53154$. According to

$$
\frac{1}{\mathrm{f}}=(\mathrm{n}-1)^{*}\left(\frac{1}{\mathrm{r}_{1}}-\frac{1}{\mathrm{r}_{2}}\right)-\frac{(\mathrm{n}-1) \times d}{\mathrm{n} * \mathrm{r}_{1} \mathrm{r}_{2}}
$$

( $n$ is the index of refraction of wavelength on the glass; $d$ is the thickness of the single lens; $r_{1}$, $r_{2}$ is single lens curvature radius) [4] Where $n=n_{1}=1.51432, r_{1}=-r_{2}, d=6.8 \mathrm{~mm}$, for $\mathrm{f} 1=31.86137724$ $\mathrm{mm}$. 'because $v=1.03310141 \mathrm{~mm}$, the focal length of the corresponding wavelength $\lambda_{2} \mathrm{f}_{2}=$ $30.82827586 \mathrm{~mm}$, and the corresponding wavelength in the refractive index of $\mathrm{K} 9$ glass: $\mathrm{n}_{2}=$ 1.53154 , according to the wavelength and the refractive index of diagram, $\lambda_{2}=396 \mathrm{~nm}$.

(2)After are part of a circuit and this is also our main research part. Circuit part of the main function is CMOS image information acquisition and exposure control. To realize the high-speed image acquisition, adopted FPGA as Cyclone IV as the master control chip. Using high-speed SDRAM ping-pong image cache, and then by high-speed USB2.0 communication chips, according to the data back to the computer processing circuit parts mainly divides into the FPGA image acquisition and USB image transmission parts. 

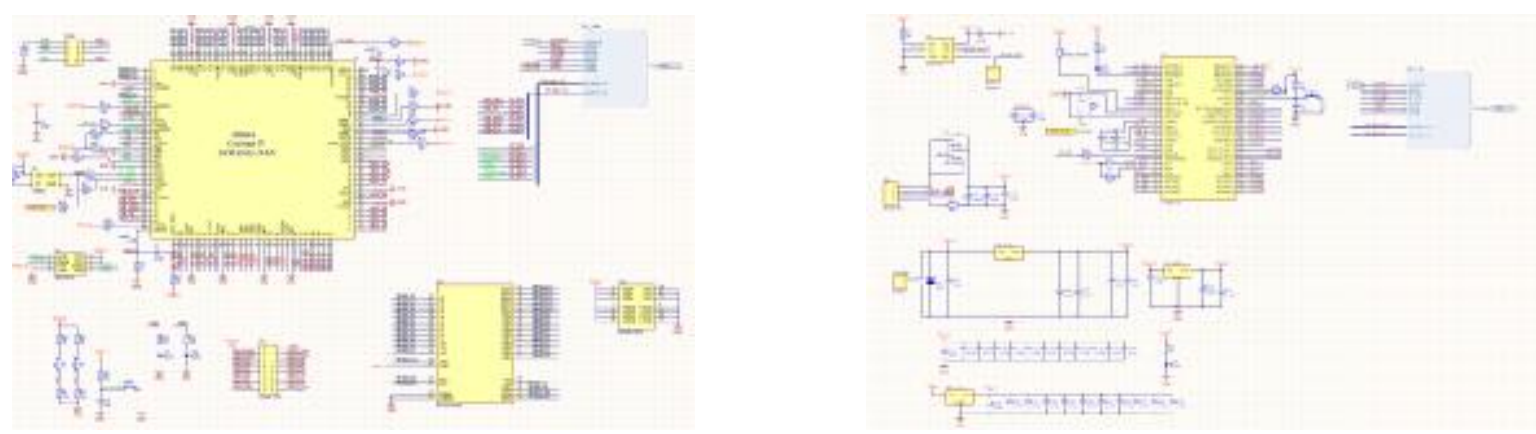

Fig. 3 circuit principle diagram

(1) The FPGA image acquisition part

The FPGA chips we use is Cyclone IV EP4CE6E22C8 series chip of Altera company. The chip inside have $6272 \mathrm{LE}$ unit. Top speed of $200 \mathrm{MHZ}$. And the highest speed of CMOS is $48 \mathrm{MHZ}$, so the chips enough to meet the needs of high-speed data acquisition. $24 \mathrm{MHZ}$ system adopts active crystal to cheer for the clock source, and then through the internal PLL frequency doubling for cmos and sdram clock.

SDRAM is used MT48LC8M16,capacity for 128 MBB (2 Meg x 16 x 4 Banks), the highest reading and writing the clock is $133 \mathrm{MHZ}$, can read and write speed to realize the ping-pong cache video data, in order to realize the purpose of the high-speed image data acquisition.

Exposure Settings using the button real-time control scheme. Convenient to test and adjust.

(2) USB image transmission parts.

USB drive circuit adopts USB drive chip CY7C68013, it integrates USB transceiver, SIE (serial interface engine), enhanced 8051 microcontroller and programmable peripheral interface. FX2 this ingenious structure can make the data transfer rate reach $56 \mathrm{MB} / \mathrm{s}$, namely the USB2.0 allow maximum bandwidth, in FX2, intelligent SIE hardware can handle many USB1.1 and USB2.0 protocol, so as to ensure the compatibility of USB. GPIF (General Programmable Interface) and SlaveFIFO) (8 bit or 16 bit data bus for the ATA, UTOPLA, EPP, DSP and PCMCIA Interface provides a simple and seamless connection.

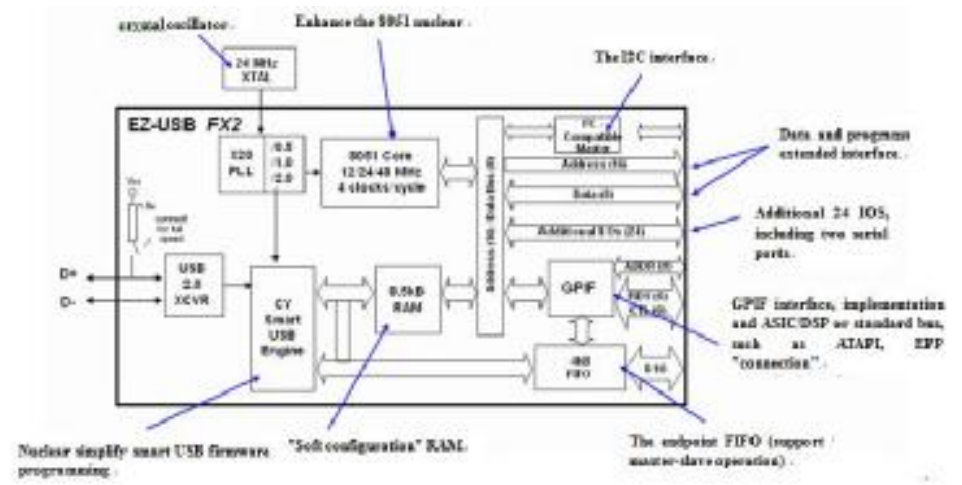

Fig. 4 The frame of CY7C68013 chip

This system mainly apply CYUSB SlaveFIFO mode to implement high-speed data upload images, realize the real-time display of the image data. In this mode the highest transmission rate is above $30 \mathrm{MB} \backslash \mathrm{S}$, and fully meet the 1280 x1024 30 BPS real-time processing of image data to display.

Main circuit procedure is the FPGA program and CY7C68013, including the FPGA program for the Verilog hardware description language, compiled by Altera corporation Quartus II software. And CY7C68013 program is written by C51 program, compiled for the keil software, through the

(3) Software part adopts a key to "prison break" control, is a key to the whole process of operation, simple operation, a key control. In LabVIEW software design is called interconnection in 
the interface call library functions in the library and the executable program. Calls a DLL function, and then control the CMOS camera.

In addition, the LabVIEW calling MATLAB nodes, for the collection of image data, image processing. MATLAB image processing, the calling function imadjust, histeq, imsharpen, adapthisteq, imhistmatch, decorrstretch functions such as basic image processing. Including grayscale processing, histogram specification, sharpen, adaptive histogram and histogram matching, and contrast stretching method, make the image can achieve the effect of.

\section{Experimental results and discussion}

We used to make the experiment device and program, double color single lens high-definition imaging system is constructed. Using the standard test card to 0.5 times ISO12233 system imaging, and the imaging quality of visual degree and Imatest software use objective score. Test results as shown in the figure below:

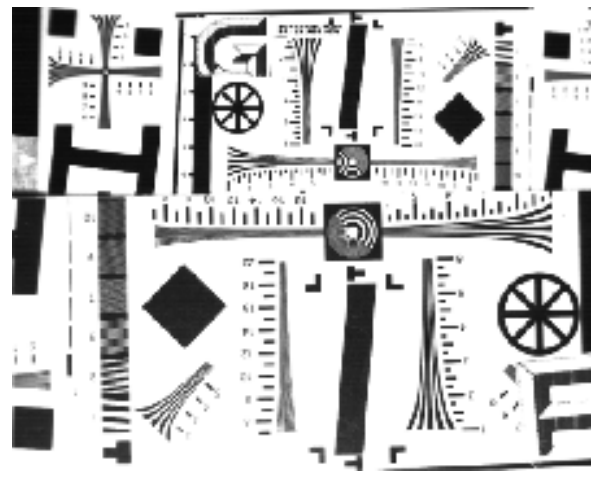

Fig. 5 Shooting figure

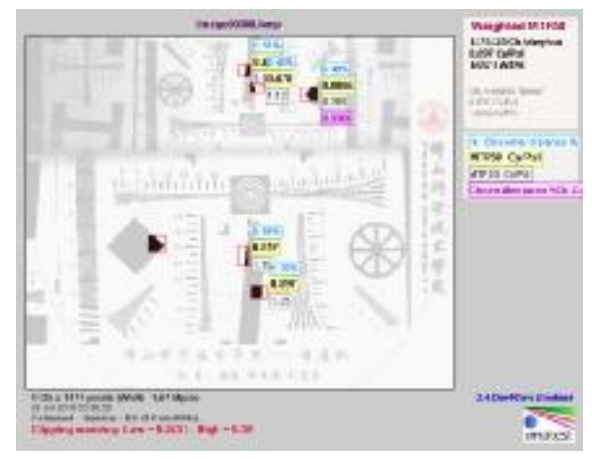

Fig. 6 Software evaluation results

Table 1 Score calculation results

\begin{tabular}{|c|c|c|c|c|c|c|c|c|}
\hline test card & wedge & multiple & $\begin{array}{c}\text { Visual } \\
\text { reading }\end{array}$ & 0.3 & $\begin{array}{c}\text { image } \\
\text { height }\end{array}$ & length & distinguishability & Data rate \\
\hline far & across & 100 & 8 & 0.3 & 1280 & 270 & 1137.778 & MAX \\
\hline & vertical & 100 & 8 & 0.3 & 1024 & 270 & 910.2222 & 2133.333 \\
\hline near & across & 100 & 12 & 0.3 & 1280 & 270 & 1706.667 & MIN \\
\hline & vertical & 100 & 15 & 0.3 & 1024 & 270 & 1706.667 & 796.4444 \\
\hline far & across & 100 & 8 & 0.3 & 1280 & 270 & 1137.778 & AVE \\
\hline & vertical & 100 & 8 & 0.3 & 1024 & 270 & 910.2222 & 1317.333 \\
\hline near & across & 100 & 12 & 0.3 & 1280 & 270 & 1706.667 & \\
\hline & vertical & 100 & 15 & 0.3 & 1024 & 270 & 1706.667 & \\
\hline far & across & 100 & 7 & 0.3 & 1280 & 270 & 995.5556 & \\
\hline & vertical & 100 & 7 & 0.3 & 1024 & 270 & 796.4444 & \\
\hline near & across & 100 & 12 & 0.3 & 1280 & 270 & 1706.667 & \\
\hline & vertical & 100 & 11 & 0.3 & 1024 & 270 & 1251.556 & \\
\hline far & across & 100 & 7 & 0.3 & 1280 & 270 & 995.5556 & \\
\hline & vertical & 100 & 8 & 0.3 & 1024 & 270 & 910.2222 & \\
\hline near & across & 100 & 15 & 0.3 & 1280 & 270 & 2133.333 & \\
\hline & vertical & 100 & 12 & 0.3 & 1024 & 270 & 1365.333 & \\
\hline
\end{tabular}




\section{Conclusion}

For single lens imaging system of imaging surface due to the existence of large depth of field from the focal problems, we build the double light single lens of high-definition imaging system experiment.The results show that the method of using double color light to illuminate objects can effectively compensate the depth of field to solve the problem of defocus.

\section{References}

[1] X .Zhong. Modern optics [M]. Beijing publishing house, 2008.1 3436-400

[2] D.H li, Y.P .Cao, Q-C.Zhang, Q.H. Wang. Modern engineering optics [M]. Science press. 2013.1 5, 58-63-63-63

[3] L.Li , L.S.An,Q.C. Li, Y.F.Huang . Applied optics (fourth edition) [M]. Beijing institute of technology press. 2010.1-11, 14-42149-152

[4] C. Liu, Z.M. zhao, M.Yu. Applied optics [M]. Beijing: mechanical industry publishing house. 2010

[5] H. Sun. Based on ARM and FPGA embedded high-speed image acquisition storage system [J]. Journal of electronic science and technology. 2009 (02) 